\title{
Influence of Calculation Domain Size on Numerical Simulation Results for Complex Terrain Wind Fields
}

\author{
Lian Shen ${ }^{1,2, *}$, Chenglong Wei ${ }^{1}$, Chunsheng Cai ${ }^{4}$ and Xiaoyan $\mathrm{Liu}^{3}$ \\ ${ }^{1}$ College of Civil Engineering, Changsha University, Changsha 410022, China \\ ${ }^{2}$ College of Civil Engineering, Hunan University, Changsha, 410082, China \\ ${ }^{3}$ Department of Civil and Environmental Engineering, Louisiana State University, Baton Rouge, LA, 70803, United State \\ ${ }^{4}$ College of Civil Engineering, Changsha University of Science and Technology, Changsha, 410076, China
}

Received 21 February 2019; Accepted 2 May 2019

\begin{abstract}
Computational domain size is a key factor in the numerical simulation of complex terrain wind fields. However, present studies on the selection of computational domain size in the simulation process are scarce. The influence of computational domain size on calculation results also remains unclear. This study analyzed the length and height of calculation domain and established 11 numerical wind tunnel models with different lengths to determine the influence of calculation domain size on the simulation results of complex wind fields, this. Information about the average and fluctuating wind fields of the target location was obtained using large eddy simulation. A detailed analysis was conducted on an actual mountainous terrain wind field in Zhangjiajie, China. Results show that the stable position of wind speed in the height direction is 7 to 8 times the height of the highest mountain in the simulation of complex terrain wind field. In the horizontal direction, the complex mountain topography of several kilometers cannot sufficiently generate the average wind field, and fluctuating wind fields are consistent with the actual situation. The application of additional reasonable average wind speed and fluctuating wind speed at the inlet boundary is necessary for detailed analysis of complex terrain wind fields. This study can provide a reference for numerical simulation of complex terrain wind fields.
\end{abstract}

Keywords: Inlet boundary, Numerical simulation, Complex morphology, Wind field characteristic, Computation domain

\section{Introduction}

Computational fluid dynamics (CFD) theory recently improved with the rapid development of computer technology. CFD numerical simulation technology is widely used in wind environment of complex morphology. CFD numerical simulations have many advantages, such as low cost, repeatability, and good capture of the detailed distribution of flow fields compared with field measurement and wind tunnel test. Some scholars favor this numerical simulation [1-4].

However, numerous studies [3-6] have generally focused on turbulence models and boundary conditions. Target research that concentrates on calculation domain size and its selection is lacking. Calculation domain size is also directly related to computational efficiency. The computational domain can be divided into global, regional, urban, and micro scales. The microscale includes community, street, and single building scales [7]. The global scale refers to the entire scale of the Earth and it aims to address global climate issues, such as ozone hole and greenhouse effect. The regional scale refers to the area of hundreds of kilometers, including cities, which belongs to the mesoscale category and has been widely used in meteorology. The city scale is generally $10-20 \mathrm{~km}$ and is applied to micrometeorological studies and wind energy assessment. The present study is tens of kilometers of complex terrain.

Scholars are concerned with computational theory and method of numerical simulation for complex terrain wind fields, and previous studies have achieved numerous results. Meanwhile, modeling techniques of complex computational domain are relatively weak and have not drawn the attention of researchers. Model establishment accounts for a large part in the entire process of calculating a complex CFD model and is one of the most critical issues in the research cycle. Computational resources will be over consumed if the calculation domain is excessively large. Meanwhile, the detailed flow field information around the target building will not be obtained if the calculation domain is extremely small. Obtaining the influence of the calculation domain during wind field simulation and determining the appropriate calculation domain size are the keys to numerical simulation. Therefore, this study selected the computation domain size for the numerical simulation of a wind field in complex morphology as the research background. This study also established 11 numerical wind tunnel models with different calculation domain lengths. The average and turbulence wind fields of the target position were monitored. Moreover, the mountain terrain wind field in Zhangjiajie, China was thoroughly analyzed to obtain the optimal calculation domain size of the wind field simulation. This analysis provides reference to future research on CFD numerical simulation. 


\section{State of the art}

Scholars worldwide have studied turbulence models and boundary conditions of numerical simulation of complex terrain wind fields. The Reynolds averaged Navier-Stokes (RANS) model is the mainstream turbulence model due to the limitation of early computer hardware. Tang et al. [8] used the standard RANS turbulence model to evaluate complex terrain wind resources; however, the accuracy of the wind field was relatively low. The turbulence model slowly transitioned into large eddy simulation (LES) to improve accuracy. Gousseau et al. [9] simulated the pollutant diffusion in downtown Montreal, Canada using two turbulence models, namely, RANS and LES. They compared the results of wind tunnel test and found that the calculation results of LES were obviously better than those of RANS. Uchida [10] simulated the turbulent wind field of complex mountain topography by using the LES method. Jiang et al. [11] summarized the progress of LES in the study of atmospheric boundary layer in the past 30 years. They indicated that the uniqueness of LES and its simulation characteristics could reveal the basic laws of random turbulence. Maurizi et al. [12] focused on inlet boundary and used one-tenth of slope as the airflow transition section to analyze the mountainous terrain of a $14 \mathrm{~km} \times 15 \mathrm{~km}$ terrain area; however, the slope's practicability needs to be improved further. Hu et al. [13] treated the boundary of a mountain canyon with the curve transition section; however, the artificial wind attack angle was generated, which directly affected the distribution of the target wind field. Li et al. [14], Ti [15], Yu [16], and Yao et al. [17] studied the wind field of a mountain canyon bridge site. These scholars reached numerous conclusions but did not provide references for the calculation domain size of the numerical model. Many scholars have explored the coupling technique between weather research forecasting (WRF) model and CFD software [18-19]. Baik et al. [20] coupled the RANS model with a mesoscale model to study Seoul's atmospheric flow and pollutant diffusion. Liu et al. [21] used WRF and LES to couple a multiscale analysis of traffic pollution in a district in Beijing. Xie [22] reported that the coupling method of LES and mesoscale meteorological models is the direction of the numerical simulation of urban communities. However, the workload of this simulation model was large, and considerable computational resources were needed to be consumed with low computational efficiency. At present, related reports on the principle of size selection of coupled computational domain are especially rare.

The results of previous studies are mainly for turbulence models and other issues, such as inlet boundary conditions. However, the study on calculation domain size remains scarce, especially the quantitative study of the height and length of the computational domain in complex terrain simulation. To solve this problem, the present study established 11 numerical wind tunnel models with different calculation lengths. The influence law of different length calculation domains was obtained by monitoring the average wind field and turbulence of the target location. The wind field of the different heights and inlet lengths of the actual mountainous terrain in Zhangjiajie, China was thoroughly analyzed. The influence of the numerical simulation field on the simulation results was also quantitatively analyzed, which can be reference for future numerical simulations of complex wind fields.

The remainder of this study is organized as follows. Section 3 introduces the control equations of the LES and

details the standard and actual numerical models. Section 4 analyzes the simulation results of the actual wind field and obtains the general rules of the influence of the calculation domain size on the wind field and the basic principles of calculation and selection. Finally, Section 5 summarizes the study and provides relevant conclusions.

\section{Methodology}

\subsection{LES equations}

This study adopts a LES turbulence model given its rapid development. Deardoff [23], an atmospheric scientist, first applied LES turbulence model in engineering to decompose each variable into a solvable scale $\bar{\phi}$ and an unsolvable scale $\phi^{\prime}$ by a filter function. The solvable scale $\bar{\phi}$ can be expressed as

$$
\bar{\phi}=\int_{D} \phi^{\prime} G\left(x, x^{\prime}\right) d x^{\prime}
$$

where $D$ is the flow area; $x^{\prime}$ is the spatial coordinate inside the actual flow area; $x$ is the filtered large-scale space coordinate; and $G\left(x, x^{\prime}\right)$ is a filtering function, which can be expressed as follows:

$$
G\left(x, x^{\prime}\right)= \begin{cases}\frac{1}{V}, & x \in v \\ 0, & x \notin v\end{cases}
$$

where $\mathrm{V}$ is the size of the geometric space occupied by the control volume, and the spatial Navier-Stokes (N-S) equation under the filtered transient can be expressed as:

$$
\begin{aligned}
& \frac{\partial \bar{u}_{i}}{\partial x_{i}}=0 \\
& \frac{\partial \bar{u}_{i}}{\partial t}+\frac{\partial \bar{u}_{i} \bar{u}_{j}}{\partial x_{j}}=-\frac{1}{\rho} \frac{\partial \bar{p}}{\partial x_{i}}+\frac{\partial}{\partial x_{j}}\left[\left(v \frac{\partial \bar{u}_{i}}{\partial x_{j}}+\frac{\partial \bar{u}_{j}}{\partial x_{i}}\right)+\tau_{i j}\right]
\end{aligned}
$$

Formulas (3) and (4) are the momentum conservation and mass conservation equations, respectively, wherein the amount of the horizontal line indicates the filtered solvable scale, $u$ denotes velocity, $p$ represents pressure, and $\tau_{i j}$ is the subgrid scale stress.

\subsection{Standard model and parameter settings}

Eleven CFD numerical wind tunnel models with different inlet lengths were established to analyze the flow field influence of the inlet distance on the calculation domain. The inlet distance was increased from $0.3 \mathrm{~m}$ to $3 \mathrm{~m}$. Different models had different inlet roughness lengths, as shown in Fig. 1. The numerical wind tunnel test section had a width of $2.2 \mathrm{~m}$, a height of $2 \mathrm{~m}$, and cube size of $0.1 \mathrm{~m} \times$ $0.1 \mathrm{~m} \times 0.1 \mathrm{~m}$ (length $\times$ width $\times$ height), where the spacing of each cube is $0.1 \mathrm{~m}$.

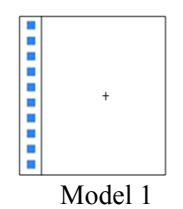

Model 1
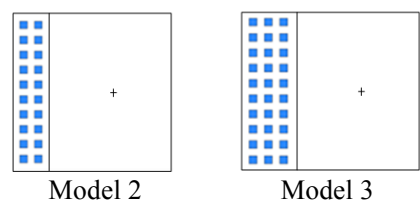


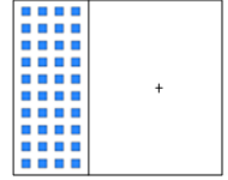

Model 4

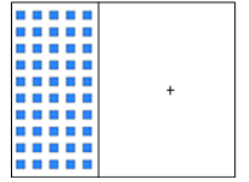

Model 5

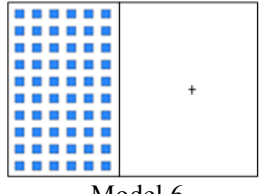

Model 6

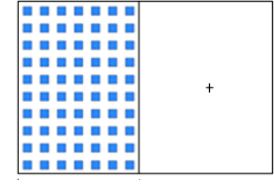

Model 7

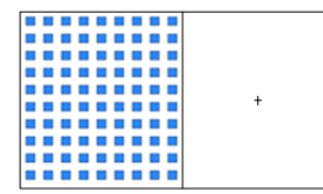

Model 9

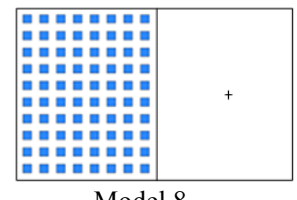

Model 8

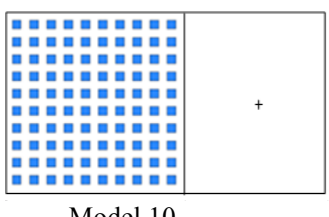

Model 10

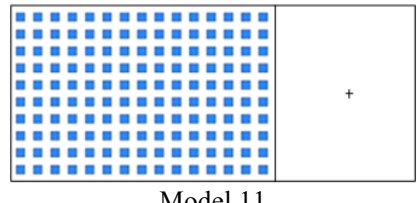

Fig. 1. Schematic of different cases

A hexahedral mesh was used for mesh generation to ensure calculation accuracy. The mesh was encrypted near the ground and the cube during generation. To satisfy the $y+$ value requirements, the height of the first-layer grid obtained by conversion was $0.0014 \mathrm{~m}$, and the height of the bottomlayer grid was $0.001 \mathrm{~m}$ in the actual process. The nearsurface grid stretching ratio was 1.1, and the grid ratio stretching away from the ground was 1.2. The number of meshes increased from 3.8 million to 8.4 million when the number of meshes met the requirements of AIJ [24]. The model and mesh diagram of Model 11 are shown in Fig. 2, where the coordinates of the monitoring center are $\mathrm{x}=1, \mathrm{y}=$ 1 , and $\mathrm{z}=0.1$.
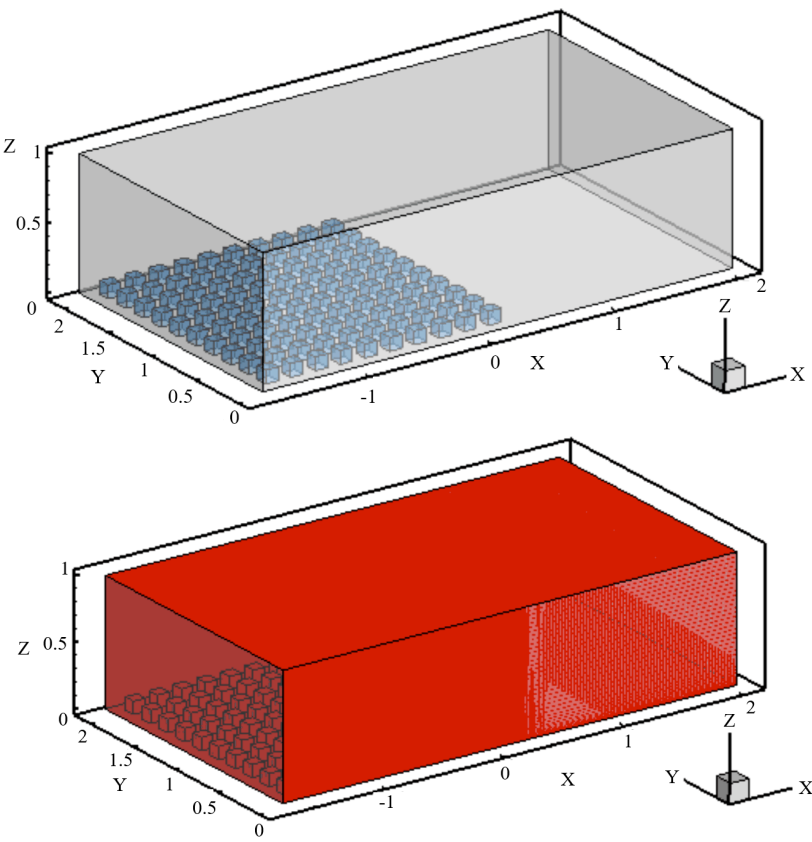

Fig. 2. Calculation model
As shown in Fig. 1, different models had different inlet roughness lengths; however, the test section size of each numerical wind tunnel was consistent with the monitoring point position. The LES turbulence model was adopted in the numerical simulation. The inlet mean wind speed was 10 $\mathrm{m} / \mathrm{s}$; the earth surface, the top surface, the side, and the outlet adopted nonslip, free-slip, symmetrical, and pressureoutlet boundary conditions, respectively. This study solved the $\mathrm{N}-\mathrm{S}$ equation using pressure-implicit with splitting of operators method. The convection and diffusion terms were in the second-order central difference scheme. Superrelaxation method was used to solve the pressure Poisson equation. The factors of pressure and momentum relaxation were 0.3 and 0.7 , respectively.

\subsection{Verification and results}

The entire calculation process used hyper-threaded parallel computing, and Cases 1-11 represent the working conditions of Models 1-11, respectively. The velocity contour obtained by the calculation of Case 10 is shown in Fig. 3. From the figure, the wind speed had a large fluctuation near the ground, and the fluctuation far from the bottom surface position was small.

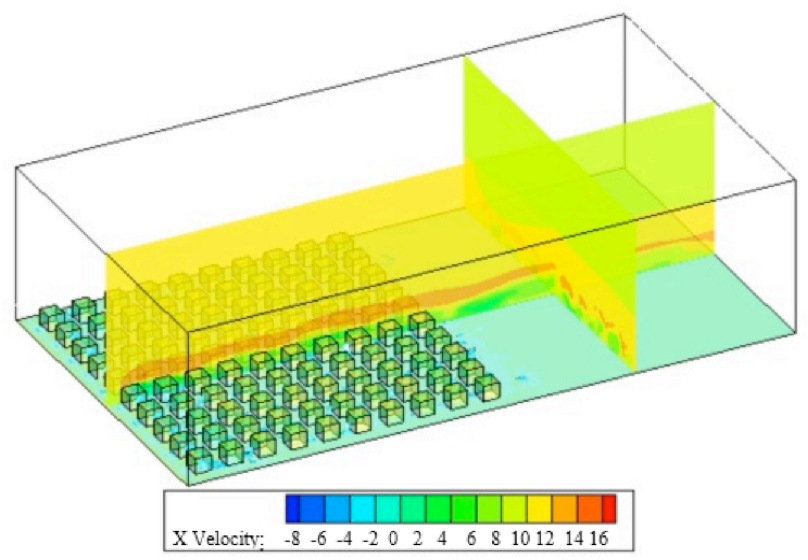

Fig. 3. Contour of calculation results

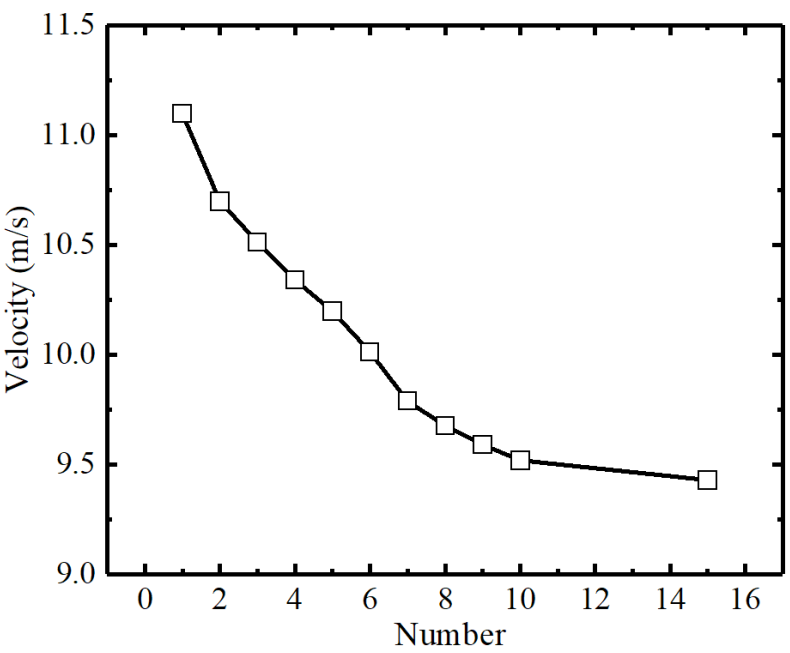

Fig. 4. Variation of average wind speed changes for different cases

The wind speed time history at different cases of the monitoring centers was analyzed. The average wind speed and turbulence intensity of the 11 cases were obtained, as shown in Figs. 4 and 5, respectively. From the figures, the average wind speed decreases with the increase of the rough length of the inlet, which indicates that the rough elements 
have a drag effect on the wind speed. The average wind speed changes significantly with the increase of the inlet distance in Cases 1-10; however, the value changes gradually after Case 10 . With sufficient rough length, the drag effect of the surface roughness on the average wind speed tends to be stable. For the turbulence intensity, the values increase with the inlet length, indicating that the cube brings a large disturbance to the wind field at the target position. Similar to the average wind speed, the average value of turbulent flow also changes significantly with the increase of the inlet distance in Cases 1-10. The value changes gradually after Case 10 . Therefore, the disturbance caused by the surface roughness tends to be stable when the rough length is sufficient.

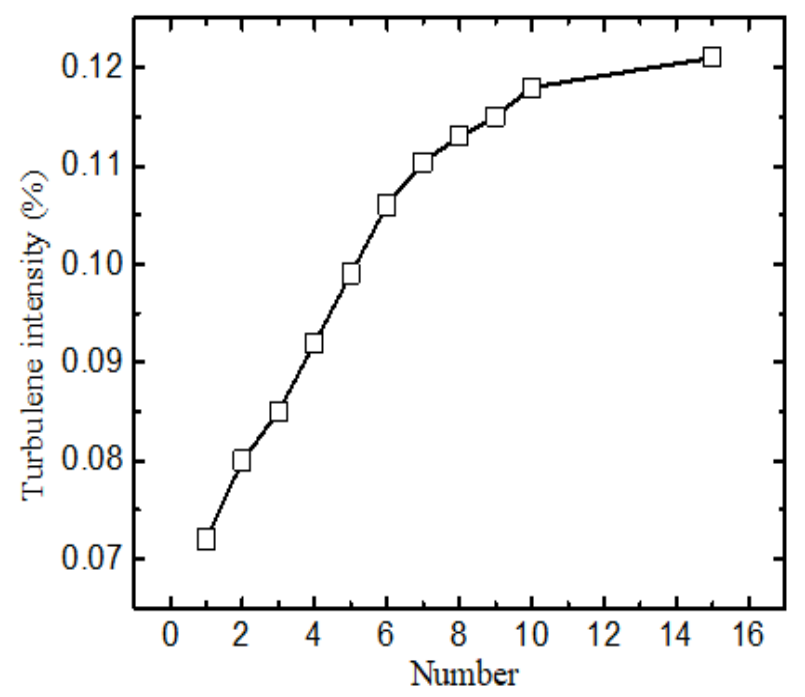

Fig. 5. Variation of turbulence intensity for different cases

The model verifies two problems. (1) First, the surface rough element has a drag effect on the wind field, and the drag force tends to be balanced when the rough distance is sufficiently long. (2) Second, the surface roughness element produces turbulence to the wind field. The turbulence tends to be balanced when the rough distance is sufficiently long. The premise of this verification is that the roughness length should be sufficiently long. This idea can be converted into the study of wind fields of mountain canyons. The rough element can be equivalent to the terrain fluctuation condition. That is, the fluctuation of the terrain makes the wind field stable within the feature height when the calculation domain is infinite. However, the calculation domain cannot be infinite in the actual CFD numerical simulation. The next section analyzes whether the mountain model can generate an atmospheric boundary layer consistent with the actual situation.

\subsection{Actual terrain geometric model}

The canyon of the Lishui Bridge in Zhangjiajie, China was taken as the research background to analyze the calculation domain height and inlet distance of the geometric model of the actual mountainous complex terrain. The mountain model adopted the actual size of the calculation area $(10 \mathrm{~km}$ $\times 9 \mathrm{~km} \times 4 \mathrm{~km}$ ), as shown in Fig. 6 . The numerical model also adopted a full hexahedral structural mesh for calculation accuracy. The mesh was encrypted near the ground. The lowest mesh height was $1 \mathrm{~m}$, and its stretching ration near the ground was 1.05. Meanwhile, the mesh further from the ground was 1.15. The total number of grids was 6752495 .
The computational grid passed the independence test during the simulation. The inlet wind profile was not processed to obtain the influence of the mountain surface on the wind field. In addition, the velocity boundary condition of the entire field was $20 \mathrm{~m} / \mathrm{s}$. The surface, the top surface, the symmetry plane, and the outlet adopted nonslip, free-slip, symmetrical, and pressure-outlet boundary conditions, respectively, as shown in Fig. 7.

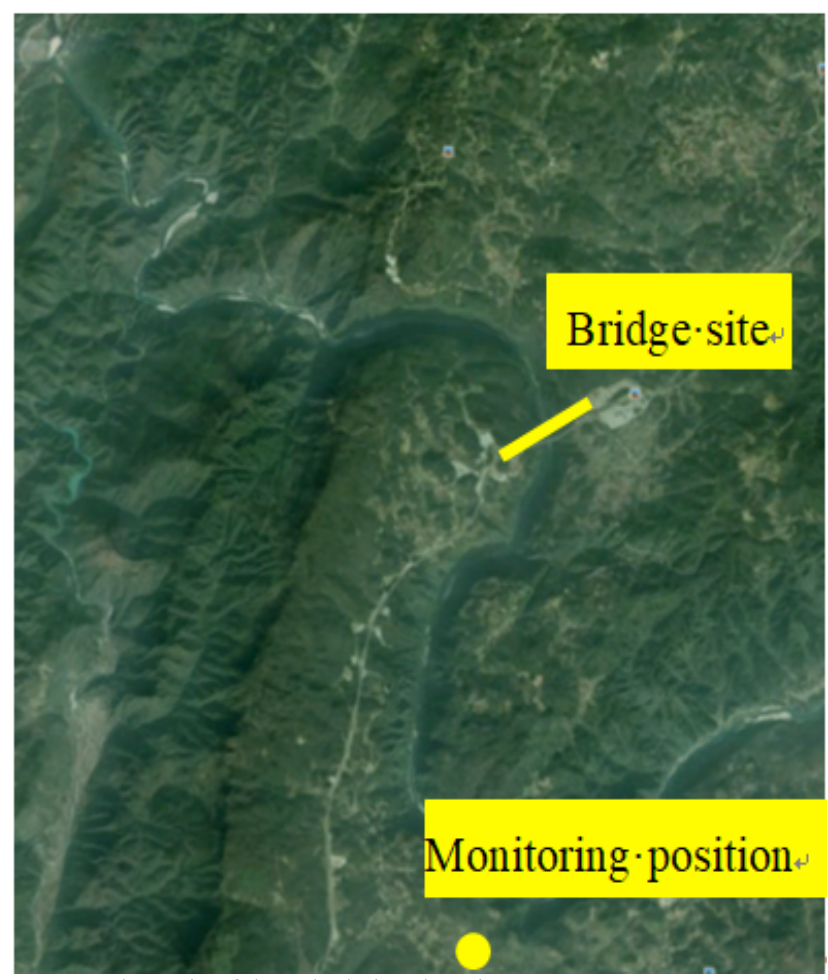

Fig. 6. Schematic of the calculation domain

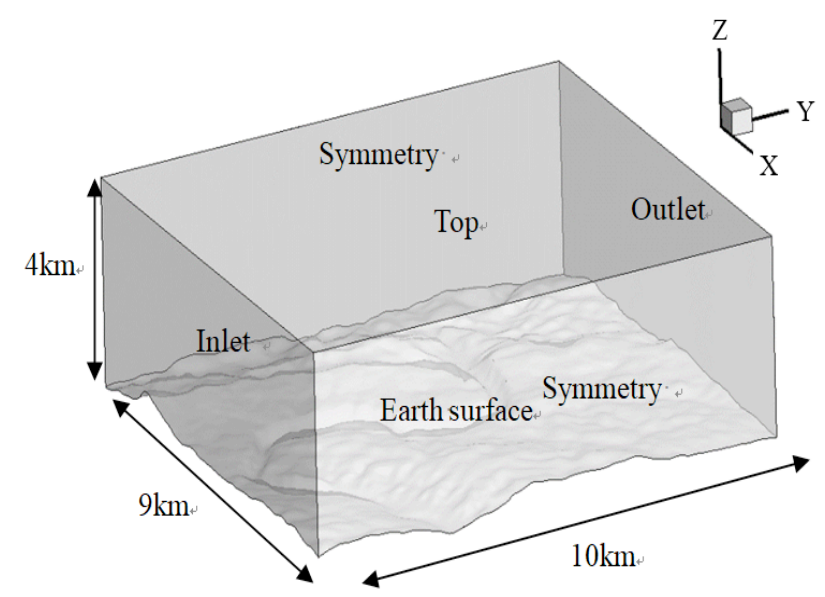

Fig. 7. Boundary condition

\section{Result analysis and discussion}

The calculated velocity contour is shown in Fig. 8. The wind velocity has an obvious fluctuation near the ground due to the influence of complex earth surface, and its maximum velocity value reaches $26.23 \mathrm{~m} / \mathrm{s}$. The wind velocity away from the ground is relatively stable, which is similar to the inlet wind speed. 


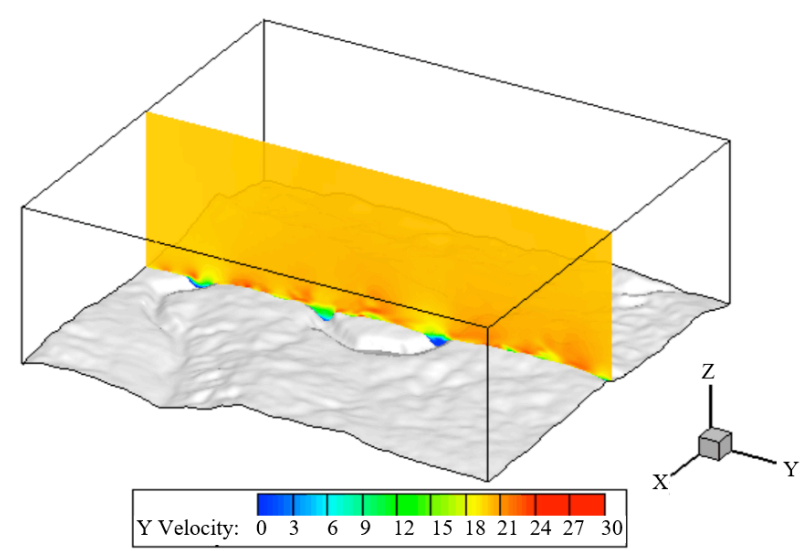

Fig. 8. Velocity contour

\subsection{Height influence of the actual model}

The average wind pressure coefficient range $\Delta C_{p}(z)$ can be used as the standard for assessing the height $(\mathrm{H})$ of the calculation domain [25], and $\Delta C_{p}(z)$ can be expressed as

$$
C_{p}=\left(p_{i}-p_{\infty}\right) /\left[0.5 \rho v_{H}^{2}\right]
$$

$$
\Delta C_{p}(z)=C_{p, \max }(z)-C_{p, \text { min }}(z)
$$

where $C_{p}$ is the average wind pressure coefficient of the monitoring point; $p_{i}$ is the static pressure; $p_{\infty}$ is the static pressure of the top surface of the calculation domain; $\rho$ is the air density; $v_{H}^{2}$ is the square of the velocity at the reference point; and $C_{p, \max }(z)$ and $C_{p, \min }(z)$ are the maximum and minimum values of the average wind pressure at height $z$, respectively, and the difference between the two coefficients is the range of the average wind pressure coefficient.

The relationship between the average pressure coefficient (that is, the difference between the minimum and maximum pressure coefficients of the same plane) and the height is expressed in Formulas (5) and (6), as shown in Fig. 9.

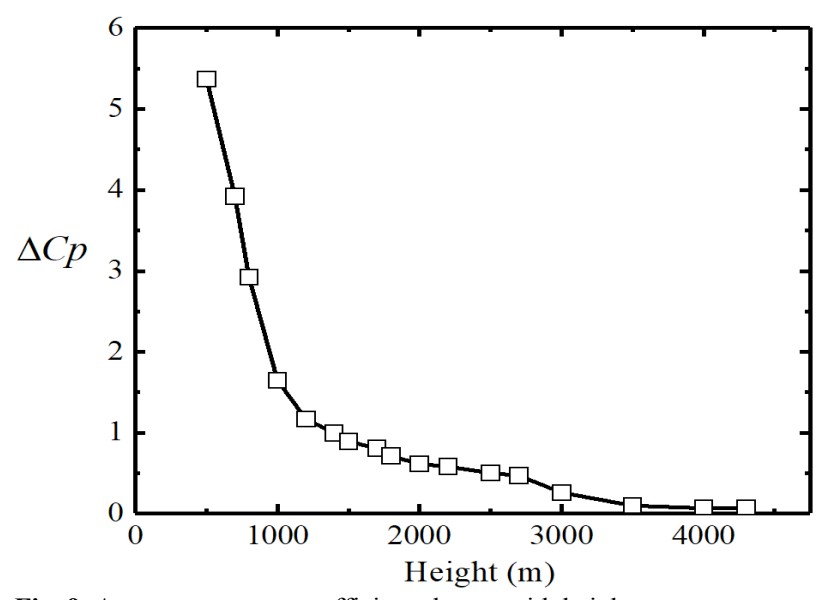

Fig. 9. Average pressure coefficient change with height

As shown in Fig. 9, the wind field is affected by the topography terrain when it is close to the ground. The wind speed fluctuates greatly; thus, the maximum and minimum pressure coefficients also deviate considerably. The wind field gradually stabilizes with the gradual increase in height, and the pressure coefficient difference in the same plane gradually becomes smaller. The pressure difference of the same plane approaches zero when the height reaches $3700 \mathrm{~m}$. This result shows that the influence of the complex surface on the wind field can be neglected. The analysis of the height of the mountain using the geometric model shows that the highest mountain elevation is $510 \mathrm{~m}$, and the stable wind speed of the height direction is $3700 \mathrm{~m}$. Therefore, obtaining 7 to 8 times of the highest mountain height is recommended for the height selection of CFD modeling of complex terrain in mountainous areas.

\subsection{Inlet distance influence of actual terrain calculation domain}

The numerical models of different inlet distances have been analyzed in Section 3.2. The results show that when the calculation domain is sufficiently long, the average wind speed and turbulence tend to be balanced after development. However, constructing an infinite computational domain is impossible due to the limitation of the number of grids and computational resources in the actual modeling process. This section discusses the wind field at different positions in the calculation domain and analyzes the influence of the horizontal distance of the model on the target wind field from a quantitative perspective. The model establishes six wind velocity observation sections. The wind velocities in the height direction were observed, and the monitoring section is shown in Fig. 10.

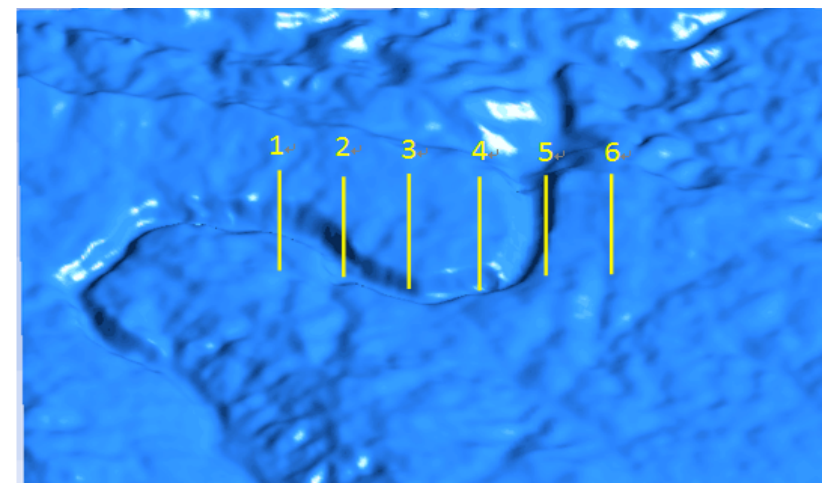

Fig. 10. Wind velocity observation points for different inlet distances

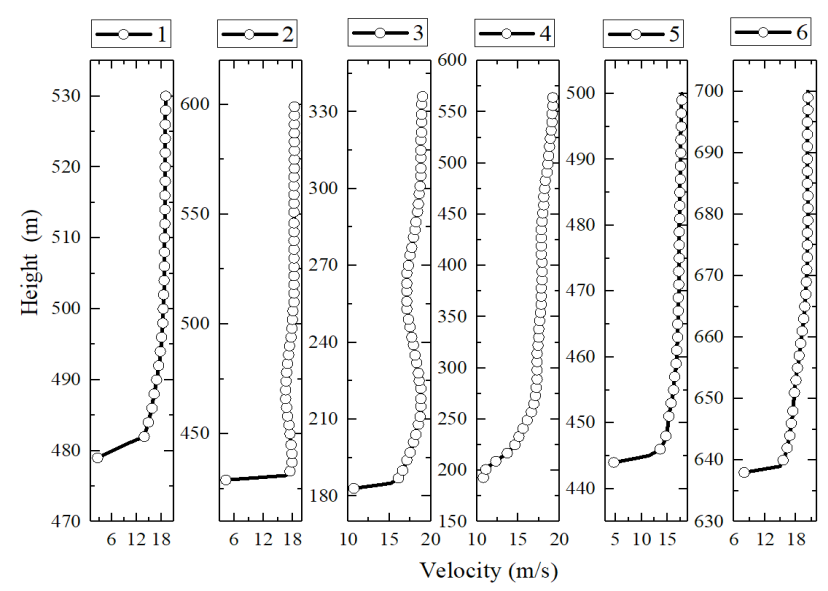

Fig. 11. Average wind profile distribution at different positions

The wind velocity time history dates of the different monitoring positions were averaged, and the average wind profile distribution of each position was obtained. The height of stable wind profile was analyzed. The stable wind velocity height was defined as the distance between the bottom of the model and the position where wind velocity changes evidently. The height distribution of stable wind 
velocity at six locations was obtained. The average wind profile and the height distribution of stable wind velocity are shown in Fig. 11.

Table 1 Height of stable wind velocity for each position (unit: $\mathrm{m}$ )

\begin{tabular}{l|l|l|l|l|l|l}
\hline Position & 1 & 2 & 3 & 4 & 5 & 6 \\
\hline Height & 18 & 85 & 150 & 360 & 32 & 21 \\
\hline
\end{tabular}

As shown in Fig. 11, the stable wind velocity is significantly higher at Positions 3 and 4 because the points are located inside the canyon, which indicates that the wind velocity profile inside the canyon is significantly different from the outside. Meanwhile, the stable wind velocity is relatively higher at Positions 2 and 5 near the canyon because the wind field at these positions is disturbed by the canyon wind field. Positions 1 and 6 are unaffected by the canyon; thus, they have relatively stable wind velocity heights of approximately $20 \mathrm{~m}$. This velocity height is far lower than the gradient wind field height (the actual gradient wind field height at mountainous area is approximately 300 $500 \mathrm{~m}$ ). The reasons for low stable wind velocity height are summarized as follows. (1) The effects of trees on the wind field were not considered in the modeling. (2) The calculation domain was extremely small, and the development of the boundary layer was still insufficient. In summary, several kilometers of mountain terrain cannot generate a reasonable atmospheric boundary layer from the perspective of average wind velocity.

The simulated wind profile was fitted with an exponential rate to analyze the wind profile index at each simulation position. The fitting result is shown in Fig. 12, and the $\alpha$ values are shown in Table 2 .

Table 2 Wind profile index $\alpha$ at each position

\begin{tabular}{l|l|l|l|l|l|l}
\hline Position & 1 & 2 & 3 & 4 & 5 & 6 \\
\hline $\boldsymbol{\alpha}$ & 0.07 & 0.026 & 0.04 & 0.11 & 0.1 & 0.24 \\
\hline
\end{tabular}

As shown in Fig. 12 and Table 2, that the value of $\alpha$ of each wind profile is considerably smaller than the class D surface value $(0.3)$ described in the standard of China (JTG/T 3360-01-2018). Therefore, the value of $\alpha$ also proves that the disturbance caused by the undulation of several kilometers of mountain cannot simulate the gradient effect of the actual wind field.

The turbulence intensity at 10-m-high position of the six monitoring profiles was analyzed to further explain the influence of the inlet distance on the wind field. The results are shown in Fig. 13.

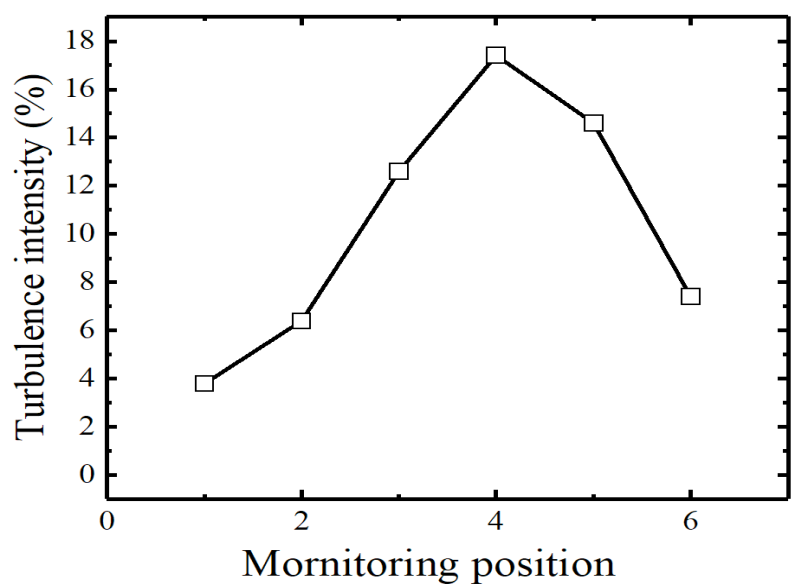

Fig. 13. Turbulence intensity at 10-m-high position
As shown in Fig. 13, the turbulence intensity in Positions $3-5$ is relatively large because these points are inside the canyon and are more complicated than the wind fields at other positions. In comparison with Fig. 8, the turbulence intensity in Fig. 13 has not stabilized after its development to a certain stage, and its value remains significantly smaller than the results from a previous study and field test. Therefore, from the perspective of turbulence intensity, the inlet boundary of several kilometers cannot generate an ideal atmospheric boundary layer.
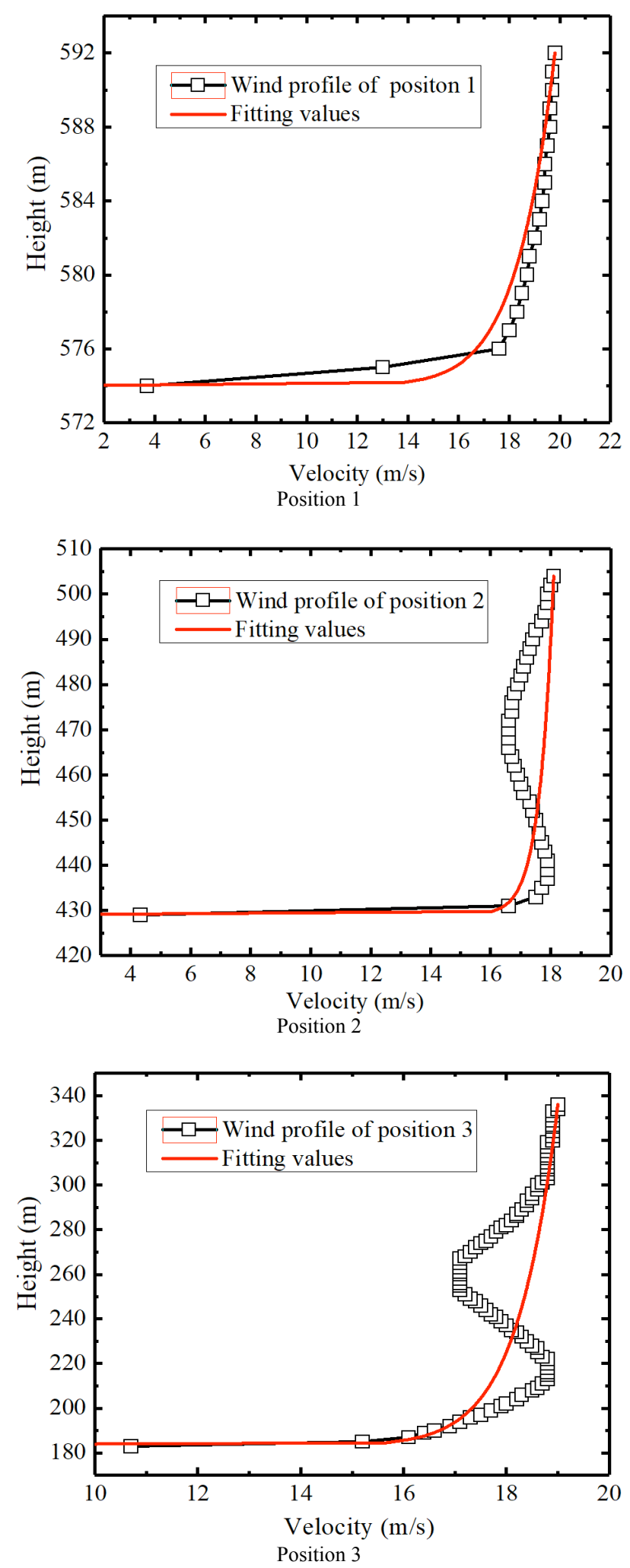

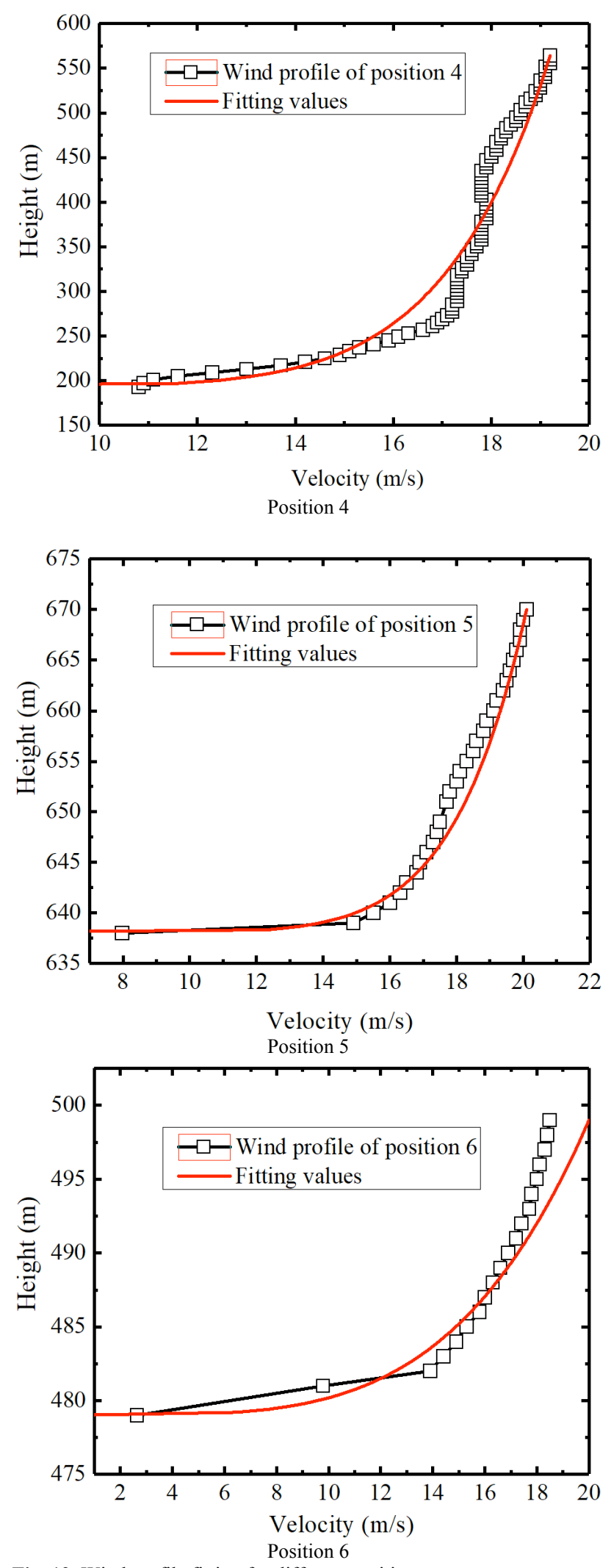

In sum, average wind speed and turbulence intensity indicate that several kilometers of mountain disturbance cannot generate an ideal atmospheric boundary layer. Thus, a suitable average wind velocity and fluctuating wind velocity at the inlet must be added to simulate complex terrain wind fields accurately.

\section{Conclusions}

This study used the LES method in investigating the wind field in a complex terrain to determine the influence of the computational domain size of complex terrain on the calculation results. The complex terrain wind field with different calculation domain lengths and heights was quantitatively analyzed. The following conclusions could be drawn from the results.

(1) The numerical wind tunnel models of 11 different inlet distances were calculated. The results show that the surface roughness has a drag effect on the wind field. The drag force tends to be balanced when the rough distance is sufficiently long. Meanwhile, the surface roughness causes a certain turbulence in the wind field, and the turbulence intensity also tends to be balanced when the rough distance is sufficiently long.

(2) The calculation domain of the complex terrain CFD model was analyzed. The analysis shows that the calculation domain can be selected from 7 to 8 times of the highest mountain elevation in the height direction.

(3) In the horizontal direction, several kilometers cannot sufficiently generate an average wind field and a fluctuating wind field consistent with the actual situation of a complex mountainous area. A reasonable average wind velocity and fluctuating wind velocity are thus required at the inlet for a refined analysis of complex terrain wind fields.

On the basis of LES, the selection of the calculation domain size of the numerical simulation in the complex wind field was analyzed in detail. Its basic selection principles, which have certain reference value for future high-efficiency wind field numerical simulations, were obtained. However, the influence of surface roughness on wind field distribution was not considered during the simulation. Therefore, the model would be further optimized after considering the surface roughness for future studies.

\section{Acknowledgments}

This work was supported by the National Science Foundation of China (No.51741802, 51808059), Hunan Provincial Natural Science Foundation of China (No.2019JJ50688), and Changsha Science and Technology Bureau Project (kc1809017).

This is an Open Access article distributed under the terms of the Creative Commons Attribution License

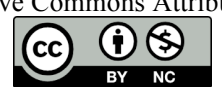

Fig. 12. Wind profile fitting for different positions

\section{References}

1. Yu J. H., Li M. S, Liao H. L. "Numerical Simulation of Effect of Mountainous Topography on Wind Field at Bridge Site". Journal of southwest jiaotong university, 51(4), 2016. Pp. 654-662.
2. Hu W. C., Yang Q. S., Yan B. W, Zhang J. "Large eddy simulation on complex terrain based on spectral element method". Engineering Mechanics (in chinese), 35(12). 2018, pp.7-14. 
3. Bilal M, Birkelund Y, Homola M, et al. "Wind over complex terrain - Microscale modeling with two types of meso-scale winds at Nygardsfjell". Renewable Energy, 99, 2016, pp. 647-653.

4. Yan B. W, Li Q. S, He Y. C, et al. "RANS simulation of neutral atmospheric boundary layer flows over complex terrain by proper imposition of boundary conditions and modification on the k-E, model". Environmental Fluid Mechanics, 16(1), 2016. pp. 1-23.

5. Liu Z, Ishihara $T$, He X, et al. "LES study on the turbulent flow fields over complex terrain covered by vegetation canopy". Journal of Wind Engineering \& Industrial Aerodynamics, 155, 2016, pp.6073.

6. Rai. R K, Berg L K,Kosovic B, et al. "Comparison of measured and numerically simulated turbulence statistics in a convective boundary layer over complex terrain". Bound-Lay Meteor, 163 (1), 2017. pp.69-89.

7. Britter R E, Hanna S R. "Flow and dispersion in urban areas". Fluid Mechanics, 35(35), 2003, pp.469-496.

8. Tang X Y, Zhao S, Fan B, et al. "Micro-scale wind resource assessment in complex terrain based on CFD coupled measurement from multiple masts". Applied Energy, 238, 2019, pp. 806-815.

9. Gousseau P, Blocken B, Stathopoulos T, et al. "CFD simulation of near-field pollutant dispersion on a high-resolution grid: A case study by LES and RANS for a building group in downtown Montreal". Atmospheric Environment, 45(2), 2010, pp. 428-438.

10. Uchida T. "Numerical Investigation of Terrain-Induced Turbulence in Complex Terrain by Large-Eddy Simulation (LES) Technique". Energies, 11(10), 2018, pp. 26-38.

11. Jiang W. M, Miao S. G. "Large Eddy Simulation and Atmospheric Boundary Layer Research-30 Years Retrospect and Prospect". Progress in Natural Science, 14(1), 2004, pp.11-19.

12. Maurizi A, Palma J M L M, Castro F A. "Numerical simulation of the atmospheric flow in a mountainous region of the North of Portugal". Journal of Wind Engineering \& Industrial Aerodynamics, 74-76(2), 1998, pp.219-228.

13. Hu P, Li Y. L, Liao H. L. "Shape of boundary transition section for mountain-gorge bridge site terrain model". Acta aerodynamic sinica, 31(2), 2013, pp. 231-238. (in Chinese)

14. Li Y. L., Cai X. T, Tang K, et al. "Study of spatial distribution feature of wind fields over bridge site with a deep-cutting gorge using numerical simulation". China Civil Engineering Journal, 44(2), 2011, pp.116-122.
15. Ti Z. L., Li Y. L., Liao H. L. "Effect of ground surface roughness on wind field over bridge site with a gorge in mountainous area". Engineering Mechanics, 34(6), 2017, pp.73-81. (in Chinese)

16. Yu J. H., Li M. S., Li S. P., et, al. "Influence of joint distribution of wind speed and wind direction and topographic effect on the design wind speed of bridge in mountainous area". China Journal of Highway and Transport, 31(8), 2018, pp.122-128.

17. Yao J F., Shen G. H, Lou W. J., et, al. "CFD-Based numerical simulation of wind field characteristics on valley and col terrain". Journal of harbin institude of technology, 48 (12), 2016, pp. 165 171.

18. Ren H, Laima S, Chen W L, et al. "Spatial correlation-based WRF observation-nudging approach in simulating regional wind field". Wind and Structures, 28(2), 2019, pp. 129-140.

19. Uraisamy V J, Dupont E, Carissimo B. "Downscaling wind energy resource from mesoscale to microscale model and data assimilating field measurements". Journal of Physics: Conference Series. IOP Publishing, 555(1), 2014, pp.012-031.

20. Baik J J, Park S B, Kim J J. "Urban Flow and Dispersion Simulation Using a CFD Model Coupled to a Mesoscale Model". Journal of Applied Meteorology \& Climatology, 48(8), 2009, pp. 1667-1681.

21. Liu Y S, Miao S G, Zhang C L, et al. "Study on micro-atmospheric environment by coupling large eddy simulation with mesoscale model". Journal of Wind Engineering \& Industrial Aerodynamics, 107-108(8), 2012, pp. 106-117.

22. Xie Z T, Castro I P. "Large-eddy Simulation For Flow And Dispersion In Urban Streets". Atmospheric Environment, 43(13), 2009, pp. 2174-2185.

23. Deardorff J W. "The Use of Subgrid Transport Equations in a Three-Dimensional Model of Atmospheric Turbulence". Journal of Fluids Engineering, 95(3), 1973, pp. 4292-438.

24. Tominaga Y, Mochida A, Yoshie R, et al. "AIJ guidelines for practical applications of CFD to pedestrian wind environment around buildings". Journal of Wind Engineering \& Industrial Aerodynamics, 96(10-11), 2008, pp.1749-1761.

25. Zhang L. L., Wu B, Yang Y, et, al. "Method of setting up the wind field of a mountainous bridge site". Journal of civil, architectural \& environment engineering, 2015(5), 2015, pp.11-17. (in chinese) 